Ivo Senjanović

Damjan Čakmak

Neven Alujević

Ivan Ćatipović

Nikola Vladimir

https://doi.org/10.21278/TOF.44201

ISSN 1333-1124

eISSN 1849-1391

\title{
RING BUCKLING ANALYSIS BASED ON THE TOROIDAL SHELL THEORY
}

\begin{abstract}
Summary
The in-plane and the out-of-plane buckling theory of rings exposed to external pressure is presented. A ring is considered as a segment of a toroidal shell. The governing ring equations are obtained by deducing the toroidal shell energy equations for the linear and the nonlinear strain. The obtained formulae for critical load are compared with those known in relevant literature. The critical load depends on the assumption concerning the load behaviour during buckling. Illustrative examples are solved numerically by means of several commercial FEM computer programs in order to investigate which assumptions are introduced in the ring buckling analysis.
\end{abstract}

Key words: $\quad$ ring buckling, energy approach, in-plane buckling, out-of-plane buckling, critical load, analytics, FEM

\section{Introduction}

Rings are ordinarily used as reinforcing structural elements in shell structures, like submarine pressure hulls, pressure vessels, and cargo tanks in liquefied gas carriers [1]-[8]. The function of rings in these structures is to sustain radial components of loading in the spanning elements, Fig. 1. These radial loads can induce high circumferential stress in the ring, which can cause buckling failure in the case of external load.

A ring is rigidly attached to the shell elements, which provides restraint on translational displacements at the joint. However, this restraint is not present in all structural components. Hence, two types of ring buckling are distinguished. When a ring is radially loaded in its plane, it may buckle by simultaneous in-plane flexure and out-of-plane flexure with torsion. If the ring has an axis of symmetry lying in its plane, the in-plane and the out-of-plane buckling are uncoupled and can be studied independently, [9].

In-plane buckling can be analysed with or without extensional effects. Critical external pressure depends on the assumption related to the pressure direction during buckling, [10]. This problem is analysed in [11]-[19]. An overview of the achieved solutions is given in [20]. 


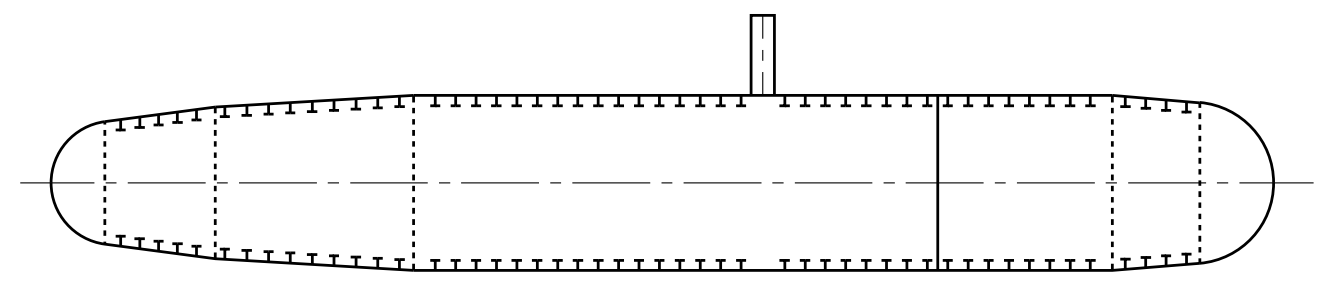

Fig. 1 Submarine pressure hull

The first solution to the problem of the out-of-plane buckling of rings was that achieved by Timoshenko and Gere, [11]. Since then, several different theories of out-of-plane ring buckling have been proposed, [21]-[24]. These theories give quite different results for some problems. A comparison and the testing of these theories are given in [25]. In addition, a new rigorous thin-walled theory for mono-symmetric rings is derived.

Recently, the vibration theory of rotating and pressurized closed toroidal shells has been developed utilizing the Rayleigh-Ritz method and a Fourier series, [26]. The same approach is then used in the development of finite strip method, which makes it possible to analyse the vibration of open toroidal shells with arbitrary boundary conditions, [27]. Moreover, rings for the in-plane and the out-of-plane vibration can be considered as segments of a toroidal shell, [28].

In this paper, the vibration theory of a toroidal shell is adapted to the in-plane and outof-plane buckling problem of rings. The obtained formulae are compared to the known results of different buckling theories. It is further investigated which assumption is used for the numerical analysis by the available FEM software.

\section{Basic expressions for the linear and the nonlinear strain energy of a toroidal shell}

The linear and the nonlinear strain energy of a toroidal shell is defined within the vibration theory of rotating and pressurized toroidal shells based on the energy approach, i.e. the Rayleigh-Ritz method, [26]. In the case of buckling analysis, the meridional displacement in the cross-sectional $\vartheta$-plane, $u$, the circumferential displacement, $v$, and the radial displacement, $w$, Fig. 2, can be assumed in the form

$$
\begin{aligned}
& u(\vartheta, \varphi)=U(\vartheta) \cos n \varphi \\
& v(\vartheta, \varphi)=V(\vartheta) \sin n \varphi \\
& w(\vartheta, \varphi)=W(\vartheta) \cos n \varphi .
\end{aligned}
$$

Functions $U(\vartheta), V(\vartheta)$ and $W(\vartheta)$ are the amplitudes of displacement which describe the cross-sectional buckling mode profile.

The linear modal strain energy, after integration in the circumferential direction, in the domain $0 \leq \varphi \leq 2 \pi$, reads

$$
\begin{aligned}
E_{s}= & \int_{9}\left[\frac{1}{2} p_{1}\left(U^{\prime}\right)^{2}+\frac{1}{2} p_{2} U^{2}+p_{3} U^{\prime} U+\frac{1}{2} p_{4}\left(V^{\prime}\right)^{2}+\frac{1}{2} p_{5} V^{2}+p_{6} V^{\prime} V\right. \\
& +p_{7} U^{\prime} V+p_{8} U V^{\prime}+p_{9} U V \\
& +\frac{1}{2} q_{1}\left(W^{\prime \prime}\right)^{2}+\frac{1}{2} q_{2}\left(W^{\prime}\right)^{2}+\frac{1}{2} q_{3} W^{2}+q_{4} W^{\prime \prime} W^{\prime}+q_{5} W^{\prime \prime} W+q_{6} W^{\prime} W \\
& +q_{7} W^{\prime \prime} U^{\prime}+q_{8}\left(W^{\prime \prime} U+W^{\prime} U^{\prime}\right)+q_{9} W^{\prime} U+q_{10} W U^{\prime}+q_{11} W U \\
& \left.+q_{12} W^{\prime \prime} V+q_{13} W^{\prime} V^{\prime}+q_{14} W^{\prime} V+q_{15} W V^{\prime}+q_{16} W V\right] \mathrm{d} \vartheta,
\end{aligned}
$$


where $p_{i}(\vartheta), i=1,2 \ldots 9$ and $q_{j}(\vartheta), j=1,2 \ldots 16$ are the variable coefficients which can be found in reference [26].
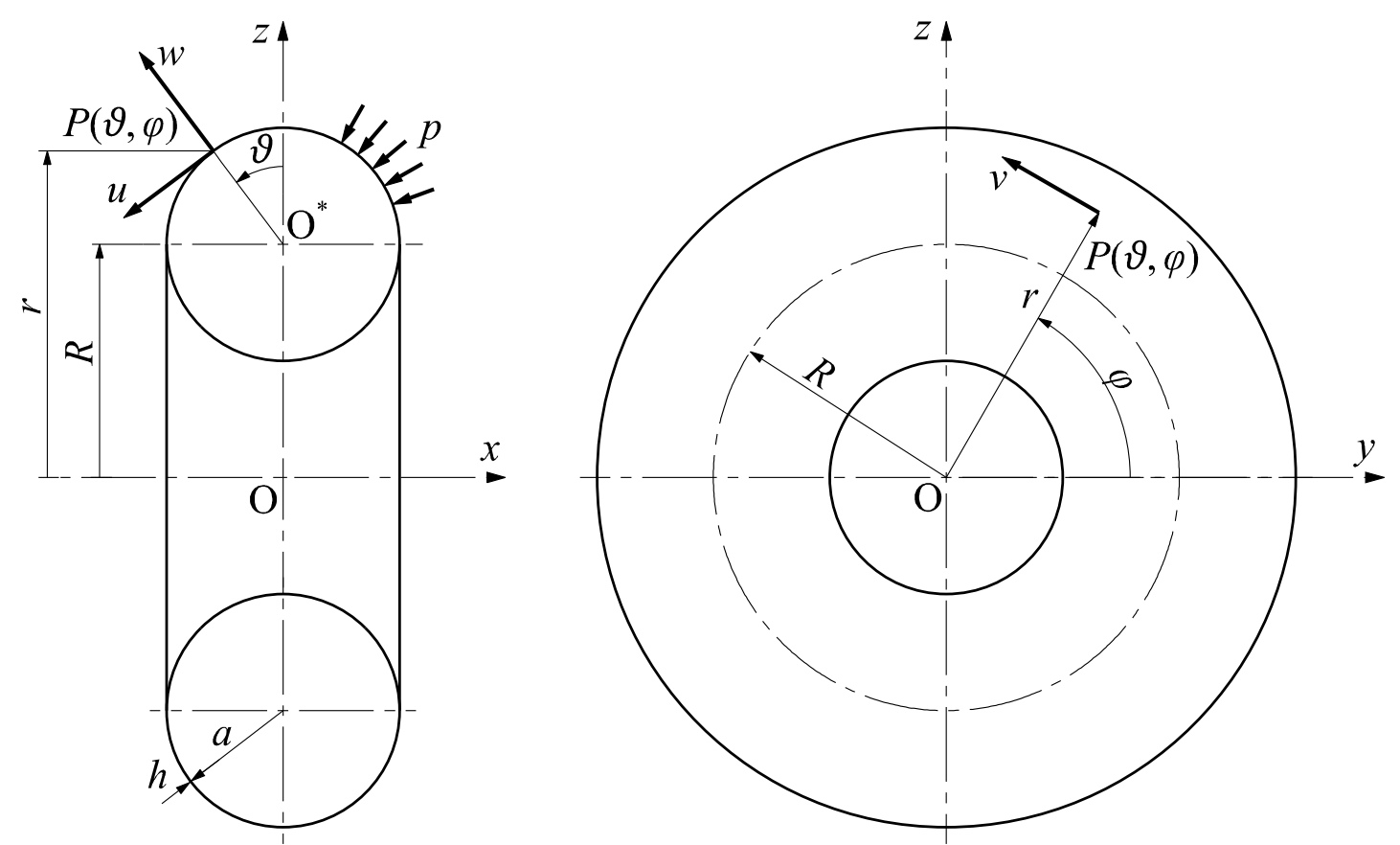

Fig. 2 Closed toroidal shell, main dimensions, load and displacements

The linearised nonlinear strain energy due to pre-stressing is obtained in the form

$$
\begin{aligned}
E_{G}= & \int_{\vartheta}\left[\frac{1}{2} c_{1}\left(U^{\prime}\right)^{2}+\frac{1}{2} c_{2} U^{2}+\frac{1}{2} c_{3}\left(V^{\prime}\right)^{2}+\frac{1}{2} c_{4} V^{2}+c_{5} V^{\prime} V\right. \\
& \left.+\frac{1}{2} c_{6}\left(W^{\prime}\right)^{2}+\frac{1}{2} c_{7} W^{2}+c_{8} U V+c_{9}\left(U^{\prime} W-U W^{\prime}\right)+c_{10} U W+c_{11} V W\right] \mathrm{d} \vartheta,
\end{aligned}
$$

where $c_{i}(\vartheta), i=1,2 \ldots 11$ are the variable coefficients which can be found in [26].

It is necessary to mention that two formulations of the nonlinear strain are known. One is the Green-Lagrange strain and the other is the engineering strain. The latter is the reduction of the former relating to the extensional terms, [29],[30].

\section{Ring in-plane buckling}

For the purpose of investigating the ring in-plane buckling, a toroidal shell segment in the vicinity of the $\vartheta=\pi / 2$ angle is considered, as shown in Fig. 3. Relevant displacements in the in-plane buckling are the circumferential and the radial buckling, $V$ and $W$. After integration, the expressions for the strain energy and the geometric strain energy, Eqs. (2) and (3) respectively, are no longer functions of the $\vartheta$ angle. Therefore, they are reduced to the following form for a unit length in the $\operatorname{arch}(b=1)$

$$
\begin{aligned}
& E_{S}=\frac{1}{2} p_{5} V^{2}+\frac{1}{2} q_{3} W^{2}+q_{16} V W \\
& E_{G}=\frac{1}{2} c_{4} V^{2}+\frac{1}{2} c_{7} W^{2}+c_{11} V W .
\end{aligned}
$$




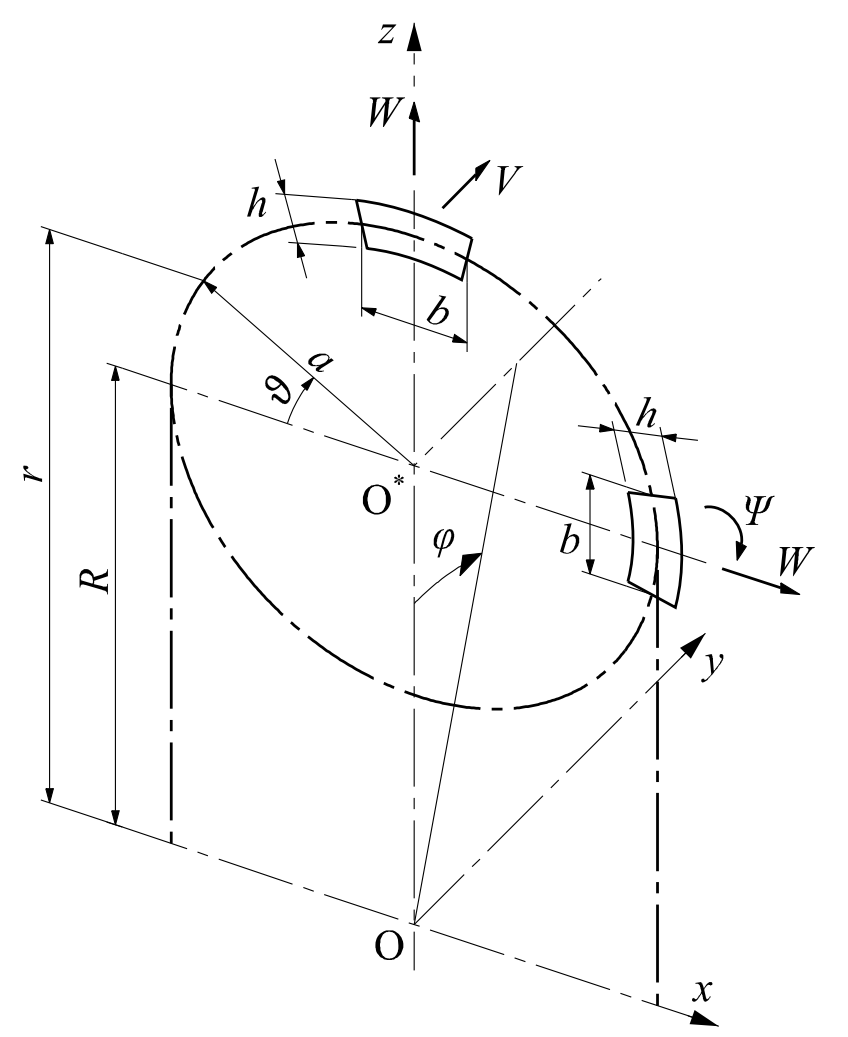

Fig. 3 Rings as segments of a toroidal shell

Coefficients $p_{i}, q_{i}$ and $c_{i}$ in Eq. (4) are specified according to [26], taking into account that $\vartheta=\pi / 2$ and $v=0$ for the ring as a one-dimensional structural element

$$
\begin{aligned}
& p_{5}=n^{2}(1+\beta) \\
& q_{3}=1+n^{4} \beta \\
& q_{16}=n\left(1+n^{2} \beta\right) \\
& c_{4}=c_{7}=\left(1+n^{2}\right) \lambda \\
& \mathrm{c}_{11}=2 n \lambda .
\end{aligned}
$$

Parameters in (5) are

$$
\beta=\frac{D}{K r^{2}}, \lambda=\frac{\tilde{N}_{\varphi}}{K},
$$

where $D=E I$ is the bending stiffness, $K=E A$ is the tensional stiffness and $\tilde{N}_{\varphi}=q r$ is the tensional force due to the pressure load $q$ per unit length. The formulae for coefficients $c_{4}, c_{7}$ and $c_{11}$ shown in (5) (taken from [28]) are related to the Green-Lagrange strains, [29].

A ring loses stability at some critical pressure load. In that moment, the ring suddenly changes its geometry, and the ordinary strain energy, $E_{S}$, is transformed into the strain energy due to pre-stressing, $E_{G}$, expressed in Eq. (4). Hence, the balance of energy reads

$$
\Pi=E_{S}-E_{G},
$$

where $\Pi$ is zero in the case of the exact solution, or it has to be minimum in the case of an approximate solution. By satisfying this condition, one obtains 


$$
\left\{\begin{array}{l}
\frac{\partial \Pi}{\partial V} \\
\frac{\partial \Pi}{\partial W}
\end{array}\right\}=\left(\left[\begin{array}{ll}
p_{5} & q_{16} \\
q_{16} & q_{3}
\end{array}\right]-\left[\begin{array}{ll}
c_{4} & c_{11} \\
c_{11} & c_{7}
\end{array}\right]\right)\left\{\begin{array}{l}
V \\
W
\end{array}\right\}=\{0\} .
$$

The first matrix in Eq. (8) is the ordinary stiffness matrix, and the second one is the geometric stiffness matrix.

The determinant of Eq. (8) has to be zero, i.e.

$$
\text { Det }=\left(p_{5}-c_{4}\right)\left(q_{3}-c_{7}\right)-\left(q_{16}-c_{11}\right)^{2}=0 \text {. }
$$

Inserting the equations from (5) into (9), after some manipulation one obtains the following quadratic equation

$$
a \lambda^{2}+b \lambda+c=0
$$

where

$$
\begin{aligned}
& a=\left(1-n^{2}\right)^{2} \\
& b=-\left(1-n^{2}\right)^{2}\left(1+n^{2} \beta\right) \\
& c=\left(1-n^{2}\right)^{2} n^{2} \beta .
\end{aligned}
$$

The solution of the eigenvalue problem from Eq. (10) reads

$$
\lambda_{1,2}=\frac{1}{2}\left[\left(1+n^{2} \beta\right) \pm\left(1-n^{2} \beta\right)\right]
$$

and one finds that

$$
\lambda_{1}=1, \quad \lambda_{2}=n^{2} \beta .
$$

Now, the determinant defined in (9) can be presented in the form

$$
\operatorname{Det}=\left(1-n^{2}\right)^{2}(\lambda-1)\left(\lambda-n^{2} \beta\right)=0 .
$$

If the mode number $n=0$, neither deformation nor buckling occurs. The value $n=1$ is related to the rigid body motion, in which case $V=W$, and there is no buckling. For the elastic modes $n \geq 2$, using (6), one finds from (13) two eigenloads

$$
\begin{aligned}
& q^{(1)}=\frac{K}{r}=\frac{E A}{r}, \\
& q^{(2)}=n^{2} \beta \frac{K}{r}=n^{2} \frac{E I}{r^{3}} .
\end{aligned}
$$

The first load, which is very high, is related to extensional buckling. The second load is relevant for bending buckling. The minimal value for $q^{(2)}$ is obtained for $n=2$, and the critical load reads

$$
q_{\mathrm{cr}}=4 \frac{E I}{r^{3}} .
$$


If the engineering strain is used [30], the coefficients of the nonlinear strain, Eq. (5), are reduced to, [28],

$$
c_{4}=\lambda, \quad c_{7}=n^{2} \lambda, \quad c_{11}=n \lambda .
$$

The determinant of the corresponding eigenvalue problem (8) is obtained in the form

$$
\text { Det }=\left(1-n^{2}\right)^{2}\left(\lambda-n^{2} \beta\right)=0 \text {. }
$$

Comparing Eqs. (14) and (19), it is obvious that the factor $\lambda-1$, related to the extensional buckling, vanishes from (19), and the critical load is the same as in the case of the Green-Lagrange strain, (17).

The buckling parameter $\mu=q_{\mathrm{cr}} r^{3} /(E I)$ determined in this paper is compared with the values known in the relevant literature, Table 1 . The value of $\mu$ depends on the assumptions made in the buckling analysis:

1. $\mu=3$ : Load remains normal to the deformed ring during buckling.

2. $\mu=3.265$ : Constant-directional load.

3. $\mu=4$ : Direction of load does not change during buckling.

4. $\mu=4.5$ : Load remains directed toward the centre of the ring during buckling.

5. $\mu=5.6$ : Constant-directional load.

In the case of a thick ring, the formula for the buckling load is extended to the following form, [31]

$$
q_{\mathrm{cr}}=\mu \frac{E I}{r^{3}} \frac{1}{1+4 \frac{E I}{G A_{S} r^{2}}},
$$

where $G=E /[2(1+v)]$ is the shear modulus and $A_{S}=k_{S} A$ is the shear area determined as a part of the ring cross-section area, $A$. The shear coefficient $k_{S}$ depends on the cross-section shape, [32],[33].

More sophisticated solutions to the problem of thick ring buckling, based on the theory of elasticity, are presented in [34]-[36].

Table 1 Ring in-plane buckling parameter $\mu=q_{\mathrm{cr}}{ }^{3} /(E I)$

\begin{tabular}{|c|c|c|c|c|c|c|}
\hline \multirow{2}{*}{} & \multirow{2}{*}{ Present } & \multicolumn{5}{|c|}{ Literature } \\
\cline { 3 - 7 } & & $(1)$ & $(2)$ & $(3)$ & $(4)$ & $(5)$ \\
\cline { 3 - 7 } & & {$[11],[12]$} & {$[12],[18]$} & {$[14]-[17],[22]$} & {$[12],[13],[22]$} & {$[19]$} \\
\hline Formula & $n^{2}$ & $n^{2}-1$ & & $n^{2}$ & & \\
\hline$n=2$ & 4 & 3 & 3.265 & 4 & 4.5 & 5.6 \\
\hline
\end{tabular}

\section{Ring out-of-plane buckling}

This type of buckling is analysed by considering the toroidal shell segment in the vicinity of the $\vartheta=\pi$ angle with two degrees of freedom, i.e. the deflection $W$ and the twist angle $\Psi$, Fig. 3. Since extensional displacements $\mathrm{U}$ and $\mathrm{V}$ are zero, the strain energy and the geometric strain energy according to Eqs. (2) and (3) are reduced to

$$
\begin{aligned}
& E_{S}=\frac{1}{2} q_{1}\left(W^{\prime \prime}\right)^{2}+\frac{1}{2} q_{2}\left(W^{\prime}\right)^{2}+\frac{1}{2} q_{3} W^{2}+q_{4} W^{\prime \prime} W^{\prime}+q_{5} W^{\prime \prime} W+q_{6} W^{\prime} W \\
& E_{G}=\frac{1}{2} c_{6}\left(W^{\prime}\right)^{2}+\frac{1}{2} c_{7} W^{2}
\end{aligned}
$$


Coefficients $q_{i}$ and $c_{i}$ in Eq. (21) are specified in [26],[28]. In the considered case $c_{6}=0$ and $c_{7}=\pi n^{2} N_{\varphi} a / r$, where $N_{\varphi}=p r$ is the circumferential membrane force per unit length due to external pressure.

The rotation angle in Eq. (21) is expressed as

$$
W^{\prime}=\frac{a}{r} X
$$

and, after some manipulation, for curvature one obtains, [26],

$$
W^{\prime \prime}=-\frac{a}{r} \frac{q_{4}}{q_{1}} X-\frac{a}{r} \frac{q_{5}}{q_{1}} W
$$

That way, the eigenvalue problem (7) for the out-of-plane buckling is reduced to

$$
\left[\begin{array}{cc}
a_{11}-b_{11} & a_{12} \\
a_{21} & a_{22}
\end{array}\right]\left\{\begin{array}{l}
W \\
X
\end{array}\right\}=\{0\},
$$

where the elements of the above matrix are given in [28]. Element $b_{11}$ contains the membrane force $N_{\varphi}$. Solving Eq. (24) for $b_{11}$ yields

$$
b_{11}=\frac{a_{11} a_{22}-a_{12} a_{21}}{a_{22}} .
$$

By exchanging the toroidal shell geometric parameters for ring parameters, as shown in [28], and by taking into account the total force $\tilde{N}_{\varphi}=N_{\varphi} b=p b r=q r$, where $b$ is the height of the ring cross-section, one obtains the following formula for eigenvalue load

$$
q=\frac{E I}{r^{3}} \frac{\left(n^{2}-1\right)^{2}}{n^{2}+(1+v)} .
$$

The critical buckling load is obtained for the first elastic mode, due to the minimum absorbed strain energy, i.e. for $n=2$

$$
q_{\mathrm{cr}}=\frac{E I}{r^{3}} \frac{9}{4+(1+v)} \text {. }
$$

Formula (27) is similar to the Timoshenko formula, [11], which can be presented in the same form

$$
q_{\mathrm{cr}}=\frac{E I}{r^{3}} \frac{9}{4+\frac{E I}{G J}},
$$

where $J=k_{\mathrm{t}} I_{\mathrm{p}}$ is the torsional modulus, as a part of the polar moment of inertia of the ring cross-section. The torsional coefficient depends on the cross-section shape, [37],[38],[42]. In the case of circular cross-section, $k_{\mathrm{t}}=2$ and formula (28) becomes identical to (27).

In literature, there are some more sophisticated buckling theories of rings with complex cross-sections which are considered, [25]. 


\section{Numerical examples}

\subsection{Ring in-plane buckling}

Since there are different analytical formulae for the critical pressure load of the ring inplane buckling, Table 1, it is interesting to consider solutions obtained by various FEM commercial computer programs. Buckling of a thin ring with the following geometric and physical properties is analysed: $r=1 \mathrm{~m}, b=0.1 \mathrm{~m}, h=0.01 \mathrm{~m}, E=2.1 \cdot 10^{11} \mathrm{~N} / \mathrm{m}^{2}, p=1 \mathrm{MPa}$ (Fig. 4a). Three software packages are used, i.e. Abaqus [39], Catia [40] and SolidWorks [41]. The ring buckling is analysed as a $1 \mathrm{D}$, a $2 \mathrm{D}$ and a 3D spatial problem, taking $v=0$. Accordingly, different beam, shell and solid finite elements are employed. The buckling modes determined by Abaqus are shown in Fig. 5. In all calculations, the critical buckling parameter $\mu$ for $n=2$ assumes a value of 3 or 4 , Table 2. Obviously, different assumptions about the load direction during buckling are applied, as explained in Section 3. Nevertheless, the buckling shapes remain similar regardless of the assumption related to the pressure direction during buckling.

a)

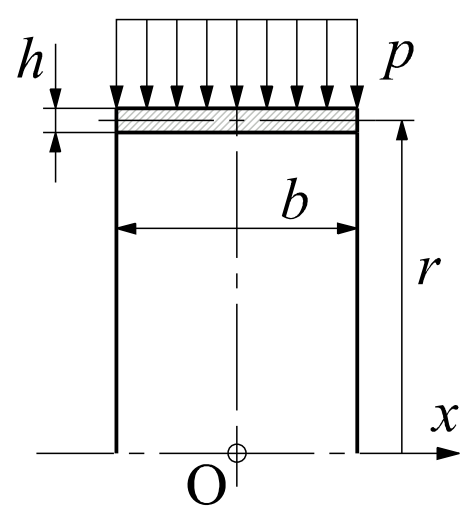

b)

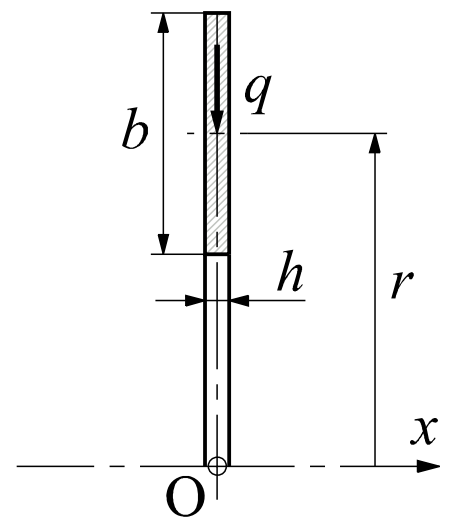

Fig. 4 Rings for: a) in-plane buckling, b) out-of-plane buckling

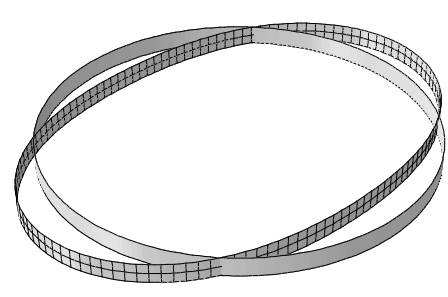

Mode 1, $n=2$

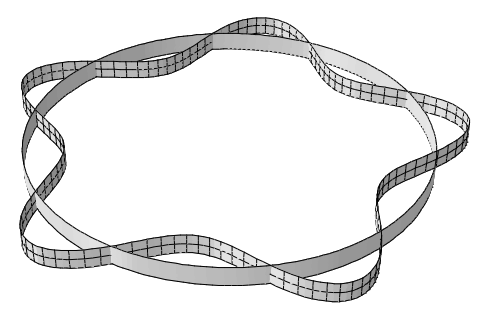

Mode 4, $n=5$

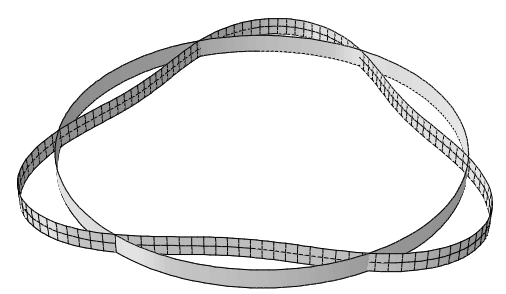

Mode 2, $n=3$

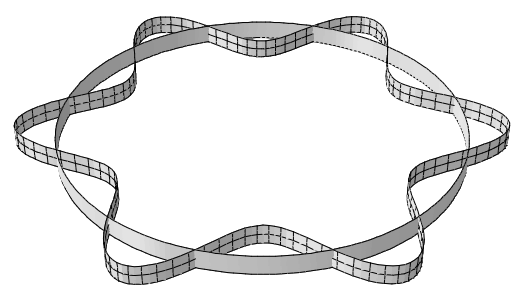

Mode 5, $n=6$

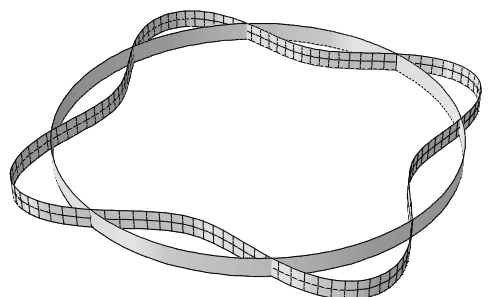

Mode 3, $n=4$

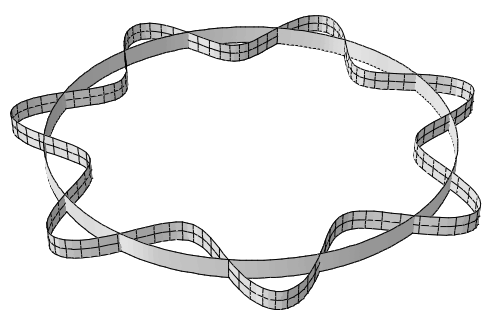

Mode 6, $n=7$

Fig. 5 In-plane buckling modes of a thin ring under external pressure (Abaqus) 
Table 2 Ring in-plane buckling parameters: $\lambda=p_{\mathrm{cr}} / p, \mu=p_{\mathrm{cr}} b r^{3} /(E I), p=1 \mathrm{MPa}$

\begin{tabular}{|c|c|c|c|c|}
\hline Software & FE type & No. FEs & $\lambda$ & $\mu$ \\
\hline \multirow{4}{*}{ Abaqus } & B32(T) & 1D, 125 & 0.052496 & 3 \\
\cline { 2 - 5 } & B33(EB) & 1D, 125 & 0.070012 & 4 \\
\cline { 2 - 5 } & S8R(nd) & 2D, 248 & 0.052495 & 3 \\
\cline { 2 - 5 } & S8R(nu) & 2D, 248 & 0.069994 & 4 \\
\cline { 2 - 5 } & C3D20R(nd) & 3D, 512 & 0.052497 & 3 \\
\cline { 2 - 5 } & C3D20R(nu) & 3D, 512 & 0.069997 & 4 \\
\hline \multirow{5}{*}{ Satia } & BEAM & 1D, 126 & 0.070031 & 4 \\
\cline { 2 - 5 } & QD8 & 2D, 252 & 0.069995 & 4 \\
\cline { 2 - 5 } & HE20 & 3D, 800 & 0.070049 & 4 \\
\hline
\end{tabular}

$\mathrm{T}$ - the Timoshenko beam theory

EB - the Euler-Bernoulli beam theory

nd - pressure normal to the deformed ring

$\mathrm{nu}$ - pressure normal to the undeformed ring

\subsection{Ring out-of-plane buckling}

Buckling of the ring shown in Fig. $4 \mathrm{~b}$ is analysed. The ring has similar geometric and physical properties as that shown in Fig. 4a: $r=1 \mathrm{~m}, b=0.1 \mathrm{~m}, h=0.01 \mathrm{~m}$, $E=2.1 \cdot 10^{11} \mathrm{~N} / \mathrm{m}^{2}, q=10^{5} \mathrm{~N} / \mathrm{m}, v=0$. Torsional modulus for the rectangular cross-section is determined according to [42], $J=k_{1} b h^{3}$, where the coefficient $k_{1}$ for the aspect ratio $b / h=10$ reads 0.312. Numerically determined buckling parameters obtained by Abaqus, Catia and SolidWorks are compared with the analytical value in Table 3. Very good agreement is observed in the cases when pressure remains normal to the undeformed ring $(\mu \approx 2)$. However, different results are obtained in the cases when pressure remains normal to the deformed ring $(\mu \approx 3)$. Buckling modes determined by Abaqus are shown in Fig. 6.

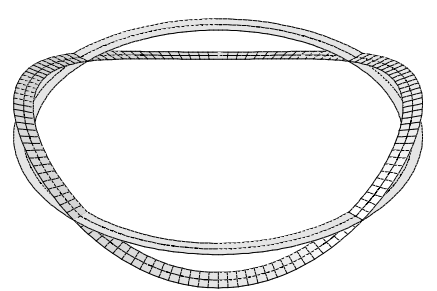

Mode 1, $n=2$

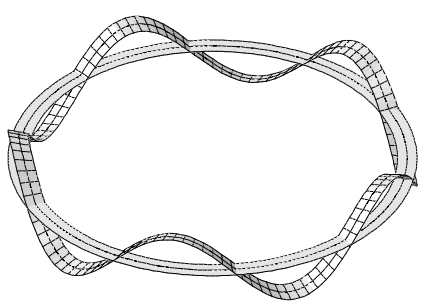

Mode 4, $n=5$

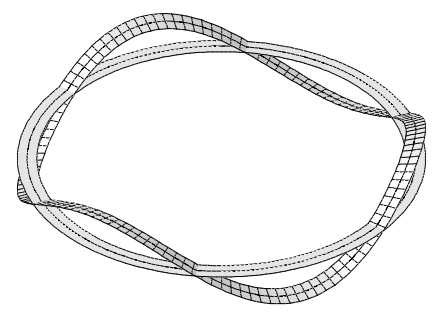

Mode 2, $n=3$

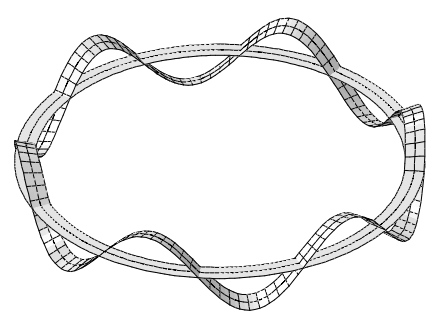

Mode 5, $n=6$

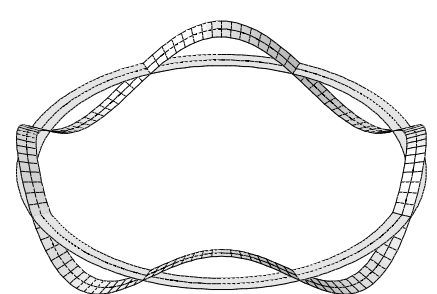

Mode 3, $n=4$

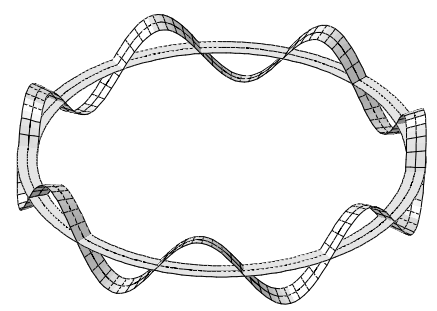

Mode 6, $n=7$

Fig. 6 Out-of-plane buckling modes of a thin ring under external pressure (Abaqus) 
Table 3 Ring out-of-plane buckling parameters: $\lambda=q_{\mathrm{cr}} / q, \mu=q_{\mathrm{cr}} r^{3} /(E I), q=10^{5} \mathrm{~N} / \mathrm{m}, v=0$, Analytical, Eq. (28): $\lambda=0.034736, \mu=1.98492$

\begin{tabular}{|c|c|c|c|c|}
\hline Software & FE type & No. FEs & $\lambda$ & $\mu$ \\
\hline \multirow{4}{*}{ Abaqus } & B32(T) & 1D, 125 & 0.050864 & 2.90653 \\
\cline { 2 - 5 } & B33(EB) & 1D, 125 & 0.034791 & 1.98807 \\
\cline { 2 - 5 } & S8R(nd) & 2D, 248 & 0.050648 & 2.89417 \\
\cline { 2 - 5 } & S8R(nu) & 2D, 256 & 0.034864 & 1.99223 \\
\cline { 2 - 5 } & C3D20R(nd) & 3D, 512 & 0.050953 & 2.91162 \\
\cline { 2 - 5 } & C3D20R(nu) & 3D, 512 & 0.034882 & 1.99326 \\
\hline \multirow{4}{*}{ Catia } & BEAM & 1D, 126 & 0.034760 & 1.98626 \\
\cline { 2 - 5 } & QD8 & 2D, 252 & 0.034861 & 1.99207 \\
\cline { 2 - 5 } & HE20 & 3D, 800 & 0.034930 & 1.99599 \\
\hline SolidWorks & SHELL6 & 2D, 536 & 0.035050 & 2.00286 \\
\hline
\end{tabular}

$\mathrm{T}-$ the Timoshenko beam theory

$\mathrm{EB}$ - the Euler-Bernoulli beam theory

nd - pressure normal to the deformed ring

$\mathrm{nu}$ - pressure normal to the undeformed ring

\section{Conclusion}

The toroidal shell theory is universal since it comprises the complete class of shells of revolution, i.e. cylindrical, conical and spherical shells, as well as circular membranes and plates. Moreover, rings for the in-plane and out-of-plane buckling analyses can be considered as segments of a toroidal shell.

By adapting the toroidal shell energy equations for the linear and the nonlinear strain to the in-plane and the out-of-plane ring buckling, a relatively simple eigenvalue problem is formulated. This leads to analytical formulae for critical loads.

In literature, there are different formulae for the ring in-plane buckling; they depend on the assumption relating to the load behaviour during buckling. The derived formulae, with the buckling coefficient $\mu=4$, correspond to the case of constant pressure direction during buckling.

For the ring out-of-plane buckling, the structure of the formulae for critical load is the same as that given by Timoshenko. However, in that formula, the appropriate torsional modulus of the considered ring cross-section has to be used.

Illustrative examples of ring buckling solved numerically by means of several commercial FEM computer programs show that different assumptions concerning the load behaviour during buckling are introduced.

For the determination of geometrical properties of rings with a very complex thinwalled cross-section, a numerical procedure based on the strip method, used for ship hull cross-sections, can be applied, [43].

\section{Acknowledgement}

This investigation has received funding within the international collaborative project Global Core Research Centre for Ships and Offshore Plants (GCRC-SOP, No. 20110030669), established by the South Korean Government (MSIP) through the National Research Foundation of South Korea (NRF). 


\section{REFERENCES}

[1] I. Senjanović: Theory of Shells of Revolution, Brodarski institut, Zagreb, 1972.

[2] E.E. Allmendinger (Ed.): Submersible Vehicle Systems Design, SNAME, Jersey City, N.J., 1990.

[3] C.T.F. Ross: Pressure Vessels Under External Pressure: Statics and Dynamics, Elsevier, London, 1990.

[4] C.T.F. Ross: Design of Submarines, University of Portsmouth, Portsmouth, U.K., 2000.

[5] J.R. MacKay: Structural Analysis and Design of Pressure Hulls: the State of the Art and Future Trends, Defence R\&D Canada - Atlantic, Technical Memorandum, DRDC Atlantic TM 2007-188.

[6] D. Štulhofer: Stress analysis of a circular cylindrical shell, reinforced by equally spaced ring frames under uniform pressure, IASS Symposium, Honolulu, Hawaii, 1971.

[7] R. Grubišić, D. Mitrov: Strength analysis of submarine pressure hull, Brodogradnja 46(1) (1998) 30-39.

[8] I. Senjanović, Z. Mravak, V. Slapničar, I. Gospić: Structure design of bilobe cargo tanks in liquefied gas carriers, Brodogradnja 50(3) (2002) 323-334.

[9] T. Wah: Buckling of thin circular rings under uniform pressure, International Journal of Solids and Structures 3(6) (1967) 967-974. https://doi.org/10.1016/0020-7683(67)90022-4

[10] Column Research Committee of Japan: Handbook of Structural Stability, Corona Publishing Company, Tokyo, 1971.

[11] S.P. Timoshenko, J.M. Gere: Theory of Elastic Stability, McGraw-Hill, New York, 1961.

[12] J. Singer, C.D. Babcock: On the buckling of rings under constant directional and centrally directed pressure, Journal of Applied Mechanics 37(1) (1970) 215-218. https://doi.org/10.1115/1.3408445

[13] A.P. Boresi: A refinement of the theory of buckling of rings under uniform pressure, Journal of Applied Mechanics 22 (1955) 95-102.

[14] G.J. Smitses: An Introduction to the Elastic Stability of Structures, Prentice-Hall, Englewood Cliffs, New Jersey, 1976.

[15] S.C. Batterman, A.I. Soler: Buckling of rings, Journal of the Engineering Mechanics Division 96(6) (1970) 1291-1296.

[16] C.E. Pearson: General theory of elastic stability, Quarterly of Applied Mathematics 14 (1956) 133-144.

[17] A.E. Armenakas, G. Herrmann: Vibrations of infinitely long cylindrical shells under initial stress, AIAA Journal 1(1) (1963) 100-106. https://doi.org/10.2514/3.1478

[18] E. Chwalla, C.F. Kollbrunner: Beiträge zum Knickproblem des Bogenträgers und des Rahmens, Stahlbau 10 (1938) 73-78.

[19] G.A. Wempner, N.E. Kesti: on the buckling of circular arches and rings, Proceedings of the Fourth U.S. National Congress of Applied Mechanics 2 (1962) 843-849.

[20] R. Schmidt: Critical constant-directional pressure on circular rings and hingeless arches, Journal of Applied Mathematics and Physics/Zeitschrift für Angewandte Mathematik und Physik (ZAMP) 31(6) (1980) 776-779. https://doi.org/10.1007/bf01594126

[21] J.E. Goldberg, J.L. Bogdanoff: Out-of-plane buckling of I-section rings, International Association for Bridges and Structural Engineering 22 (1962) 73-92.

[22] J.A. Cheney: Bending and buckling of thin-walled open section rings, J. Eng. Mech. Div., ASCE 89, EM5 (Oct. 1963) 73-92.

[23] C.H. Yoo: Flexural-torsional stability of curved beams, J. Eng. Mech. Div., ASCE 108, EM6 (Dec. 1982) 1351-1369.

[24] J.P. Papangelis, N.S. Trahair: Flexural-torsional buckling of arches, J. Struct. Eng., ASCE 113, ST4 (Apr. 1987) 889-906.

[25] J.G. Teng, J.M. Rotter: Unrestrained out-of-plane buckling of monosymmetric rings, Journal of Constructional Steel Research 7(6) (1987) 451-471. https://doi.org/10.1016/0143-974x(87)90041-1

[26] I. Senjanović, N. Alujević, I. Ćatipović, D. Čakmak, N. Vladimir: Vibration analysis of rotating toroidal shell by the Rayleigh-Ritz method and Fourier series, Engineering Structures 173 (2018) 870-891. https://doi.org/10.1016/j.engstruct.2018.07.029

[27] I. Senjanović, I. Ćatipović, N. Alujević, D. Čakmak, N. Vladimir: A finite strip for the vibration analysis of rotating toroidal shell under internal pressure, ASME Journal of Vibration and Acoustics 141(2) (2019) 021013-021013-17. https://doi.org/10.1115/1.4041734 
[28] I. Senjanović, I. Ćatipović, N. Alujević, D. Čakmak, N. Vladimir: Free in-plane and out-of-plane vibrations of rotating thin ring based on the toroidal shell theory, Archives of Mechanics, 70(5) (2018) 429-455. https://doi.org/10.1016/j.engstruct.2018.07.029

[29] M.A. Crisfield: Non-Linear Finite Element Analysis of Solids and Structures, Volume 1: Essentials, John Wiley \& Sons, Chichester, West Sussex, England, 1991.

[30] P.F. Pai, A.H. Nayfeh: A new method for the modeling of geometric nonlinearities in structures, Computers \& Structures 53(4) (1994) 877-895. https://doi.org/10.1016/0045-7949(94)90376-x

[31] J. Ratzersdorfer: Über die Stabilität des Keisringes in seiner Ebene, Zeitschrift des Österreichischen Ingenieur und Architekten Vereins 21/22 (1938) 141-148.

[32] G.R. Cowper: The shear coefficients in Timoshenko's beam theory, ASME Journal of Applied Mechanics 33(2) (1966) 335-340. https://doi.org/10.1115/1.3625046

[33] I. Senjanović, Y. Fan: The bending and shear coefficients of thin-walled girders, Thin-Walled Structures 10(1) (1990) 31-57. https://doi.org/10.1016/0263-8231(90)90004-i

[34] J.D. Renton: On the buckling of rings and long tubes of finite thickness under lateral pressure, The Quarterly Journal of Mechanics and Applied Mathematics 33(4) (1980) 435-446. https://doi.org/10.1093/qjmam/33.4.435

[35] G. Papadakis: Buckling of thick cylindrical shells under external pressure: A new analytical expression for the critical load and comparison with elasticity solutions, International Journal of Solids and Structures 45(20) (2008) 5308-5321. https://doi.org/10.1016/j.ijsolstr.2008.05.027

[36] W. Ji, A.M. Waas: The two-dimensional elasticity solution for the buckling of a thick orthotropic ring under external pressure loading, ASME Journal of Applied Mechanics 81 (2014) 011005-1-011005-12. https://doi.org/10.1115/1.4023682

[37] L. Mentrasti: Torsion of closed cross-section thin-walled beams: The influence of shearing strain, ThinWalled Structures 5(4) (1987) 277-305. https://doi.org/10.1016/0263-8231(87)90008-5

[38] I. Senjanović, Y. Fan: On torsional and warping stiffness of thin-walled girders, Thin-Walled Structures 11(3) (1991) 233-276. https://doi.org/10.1016/0263-8231(91)90003-2

[39] Dassault Systèmes: Abaqus 6.9 User's guide and theoretical manual, Hibbitt, Karlsson \& Sorensen, Inc., 2009.

[40] Dassault Systèmes: Catia V5R19 Documentation: Finite Element Reference Guide, 2007.

[41] Dassault Systèmes: SolidWorks 2012 SP5.0 Documentation, 2012.

[42] S.P. Timoshenko, J.N. Goodier: Theory of Elasticity, McGraw-Hill, New York, 1951.

[43] I. Senjanović, S. Tomašević, N. Vladimir: An advanced theory of thin-walled girders with application to ship vibrations, Marine Structures 22 (2009) 387-437. https://doi.org/10.1016/j.marstruc.2009.03.004

$\begin{array}{ll}\text { Submitted: } & 15.3 .2019 \\ \text { Accepted: } & \quad 27.11 .2019\end{array}$
Ivo Senjanović

Damjan Čakmak

Neven Alujević

Ivan Ćatipović

Nikola Vladimir

Faculty of Mechanical Engineering and

Naval Architecture, University of Zagreb,

Zagreb, Croatia 